\title{
Measurement of $\gamma$ from time-dependent analysis of
} $B_{s}^{0} \rightarrow D_{s}^{\mp} K^{ \pm}$

\author{
Agnieszka Dziurda*† \\ CERN \\ E-mail: agnieszka.dziurda@cern.ch
}

We report the measurement of the time-dependent $C P$ violating observables in $B_{s}^{0} \rightarrow D_{s}^{\mp} K^{ \pm}$ decays. The study is performed using a dataset corresponding to $3.0 \mathrm{fb}^{-1}$ of $p p$ collisions recorded in the LHCb detector in Run I of the LHC. We measure $C_{f}=0.735 \pm 0.142 \pm 0.048$, $S_{f}=-0.518 \pm 0.202 \pm 0.073, S_{\bar{f}}=-0.496 \pm 0.197 \pm 0.071, A_{f}^{\Delta \Gamma}=0.395 \pm 0.277 \pm 0.122$, $A_{\bar{f}}^{\Delta \Sigma}=0.314 \pm 0.274 \pm 0.107$, where the uncertainties are statistical and systematic, respectively. Using these observables, the CKM angle $\gamma$ is determined to be $\left(127_{-22}^{+17}\right)^{\circ}$ modulo $180^{\circ}$ at $68 \%$ $\mathrm{CL}$, where the uncertainty contains both statistical and systematic components.

9th International Workshop on the CKM Unitarity Triangle

28 November - 3 December 2016

Tata Institute for Fundamental Research (TIFR), Mumbai, India

* Speaker.

${ }^{\dagger}$ on behalf of the LHCb collaboration 


\section{Introduction}

In the Standard Model (SM) charge-parity $(C P)$ violation in weak interactions is described by a single, irreducible phase in the Cabibbo-Kobayashi-Maskawa (CKM) mixing matrix $[1,2]$. Time-dependent analyses of $B_{s}^{0} \rightarrow D_{s}^{\mp} K^{ \pm}$decays ${ }^{1}$ are sensitive to the least-well measured angle of the CKM matrix, the angle $\gamma$ equal to $\arg \left(-V_{u d} V_{u b}^{*} / V_{c d} V_{c b}^{*}\right)$. CP violation in such decays occurs due to the interference of mixing and decay amplitudes [3]. A comparison between the value of the CKM angle $\gamma$ measured from tree-level decays with the CKM parameters measured in loop-level processes provides a powerful consistency check of the SM.

The time-dependent decay rates of the $\left|B_{s}^{0}(t=0)\right\rangle$ and $\left|\bar{B}_{s}^{0}(t=0)\right\rangle$ flavour eigenstates to a final state $f$ are:

$$
\begin{aligned}
& \frac{d \Gamma_{B_{s}^{0} \rightarrow f}(t)}{d t} \propto e^{-\Gamma_{s} t}\left[\cosh \left(\frac{\Delta \Gamma_{s} t}{2}\right)+A_{f}^{\Delta \Gamma} \sinh \left(\frac{\Delta \Gamma_{s} t}{2}\right)+C_{f} \cos \left(\Delta m_{s} t\right)-S_{f} \sin \left(\Delta m_{s} t\right)\right], \\
& \frac{d \Gamma_{\bar{B}_{s}^{0} \rightarrow f}(t)}{d t} \propto e^{-\Gamma_{s} t}\left[\cosh \left(\frac{\Delta \Gamma_{s} t}{2}\right)+A_{f}^{\Delta \Gamma} \sinh \left(\frac{\Delta \Gamma_{s} t}{2}\right)-C_{f} \cos \left(\Delta m_{s} t\right)+S_{f} \sin \left(\Delta m_{s} t\right)\right] .
\end{aligned}
$$

The $C P$ violating observables in $B_{s}^{0} \rightarrow D_{s}^{\mp} K^{ \pm}$decays are dependent on the following physical parameters: the magnitude of the amplitude ratio $r_{D_{s} K} \equiv\left|A\left(\overline{B_{s}^{0}} \rightarrow D_{s}^{-} K^{+}\right) / A\left(B_{s}^{0} \rightarrow D_{s}^{-} K^{+}\right)\right|$, the strong phase difference $\delta$ and the weak phase difference $\gamma-2 \beta_{s}$. Here, the weak phase difference is a combination of the CKM angle $\gamma$ and the mixing phase $\beta_{s} \equiv \arg \left(-V_{t s} V_{t b}^{*} / V_{c s} V_{c b}^{*}\right)$ in the $B_{s}^{0}$ system. Therefore, the measurement of physical observables can be interpreted in terms of $\gamma$ or $\beta_{s}$ by using an independent measurement of the other parameter as input. For the one final state $\left(f \equiv D_{s}^{-} K^{+}\right)$, the four decay rates give five independently measureable $C P$-violating parameters:

$$
\begin{gathered}
C_{f}=\frac{1-r_{D_{S} K}^{2}}{1+r_{D_{s} K}^{2}}, \quad A_{f}^{\Delta \Gamma}=\frac{-2 r_{D_{s} K} \cos \left(\delta-\left(\gamma-2 \beta_{s}\right)\right)}{1+r_{D_{s} K}^{2}}, \quad A_{\bar{f}}^{\Delta \Gamma \Gamma}=\frac{-2 r_{D_{s} K} \cos \left(\delta+\left(\gamma-2 \beta_{s}\right)\right)}{1+r_{D_{s} K}^{2}}, \\
S_{f}=\frac{2 r_{D_{s} K} \sin \left(\delta-\left(\gamma-2 \beta_{s}\right)\right)}{1+r_{D_{s} K}^{2}}, \quad S_{\bar{f}}=\frac{-2 r_{D_{s} K} \sin \left(\delta+\left(\gamma-2 \beta_{s}\right)\right)}{1+r_{D_{s} K}^{2}} .
\end{gathered}
$$

These observables can be used to measure the CKM angle $\gamma$, with negligible [4] theoretical uncertainty.

\section{Event selection}

The measurement is based on a proton-proton collision data sample corresponding to an integrated luminosity of $3 \mathrm{fb}^{-1}$ collected by the LHCb detector during Run I period. The full detector description can be found in [5].

The $B_{s} \rightarrow D_{s}^{\mp} K^{ \pm}$decay is reconstructed using three different $D_{s}^{-}$final states: $D_{s}^{-} \rightarrow K^{-} K^{+} \pi^{-}$, $D_{s}^{-} \rightarrow K^{-} \pi^{+} \pi^{-}$, and $D_{s}^{-} \rightarrow \pi^{-} \pi^{+} \pi^{-}$. In addition, $D_{s}^{-} \rightarrow K^{-} K^{+} \pi^{-}$is split depending on the position in the Dalitz plane, into: $D_{s}^{-} \rightarrow \phi \pi^{-}, D_{s}^{-} \rightarrow K^{* 0} K^{-}$and the remaining decays. These $D_{s}^{-}$candidates are subsequently combined with a fourth particle, referred to as the "companion", to form $B_{s}^{0} \rightarrow D_{s}^{\mp} h^{ \pm}$candidates. The flavour-specific Cabibbo-favoured decay mode $B_{s}^{0} \rightarrow D_{s}^{-} \pi^{+}$ is used as a control channel. It is used in the selection optimisation as well as in studies of the decay-time acceptance and resolution and to constrain the yields of certain physics backgrounds to the $B_{s}^{0} \rightarrow D_{s}^{\mp} K^{ \pm}$decay.

\footnotetext{
${ }^{1}$ Inclusion of charge conjugate modes is implied except where explicitly stated.
} 
The different $D_{s}^{-}$final states are distinguished by using the particle identification information from LHCb's Ring Imaging Cherenkov (RICH) detector. This selection is necessarily different for each $D_{s}^{-}$decay mode and strongly suppresses cross-feed and peaking backgrounds from other misidentified decays of $b$-hadrons to $c$-hadrons. In addition, the $B_{s}^{0}$ and $D_{s}^{-}$candidates are required to be within $m\left(B_{s}^{0}\right) \in[5300,5800] \mathrm{MeV} / c^{2}$ and $m\left(D_{s}^{-}\right) \in[1930,2015] \mathrm{MeV} / c^{2}$, respectively.

\section{Multivariate fit to $B_{s}^{0} \rightarrow D_{s}^{\mp} K^{ \pm}$and $B_{s}^{0} \rightarrow D_{s}^{-} \pi^{+}$}

The signal and background component yields in the samples of $B_{s}^{0} \rightarrow D_{s}^{\mp} K^{ \pm}$and $B_{s}^{0} \rightarrow D_{s}^{-} \pi^{+}$ candidates are obtained from a three-dimensional simultaneous extended maximum likelihood fit in the $B_{s}^{0}$ mass, the $D_{s}^{-}$mass, and the log-likelihood difference $L(K / \pi)$ between the pion and kaon hypotheses for the companion particle. The total PDF for the multivariate fit is built from the product of the PDFs in each of the three fit dimensions, since correlations between the fitting variables are measured to be small in simulation.

The dominant backgrounds are related to random combinations of $D_{s}^{-}$mesons with pions or kaons, partially reconstructed decays of the type $B_{s}^{0} \rightarrow D_{s}^{-}(\pi, K)^{+} X$, and decays of $B^{0}$ and $\bar{\Lambda}_{b}^{0}$ hadrons in which the $D^{-}$or $\Lambda_{c}^{-}$candidates are misidentified as $D_{s}^{-}$candidates. Almost all background yields are left free to float, however the backgrounds whose yields are below $2 \%$ of the signal yield are fixed from known branching fractions and relative efficiencies measured using simulated events. The multivariate fit results in a signal yield of $96942 \pm 345 B_{s}^{0} \rightarrow D_{s}^{-} \pi^{+}$and $5955 \pm 90 B_{s}^{0} \rightarrow D_{s}^{\mp} K^{ \pm}$decays, shown in Fig. 1. The multivariate fit is checked for biases using large samples of data-like pseudoexperiments, and none are found.
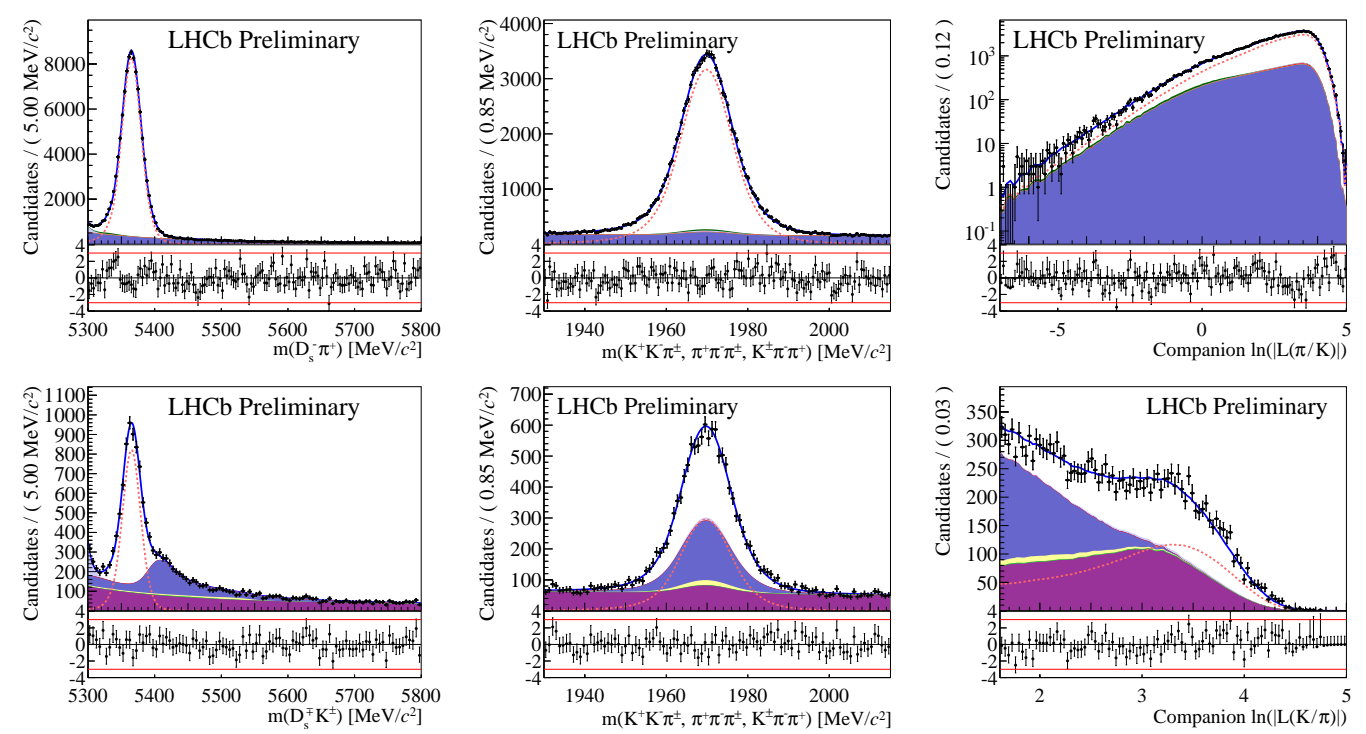

Figure 1: The multivariate fit to the (top) $B_{s}^{0} \rightarrow D_{s}^{-} \pi^{+}$and (bottom) $B_{s}^{0} \rightarrow D_{s}^{\mp} K^{ \pm}$candidates for all $D_{s}^{-}$decay modes combined. From left to right : distributions of candidates in $B_{s}^{0}$ invariant mass, $D_{s}^{-}$invariant mass, absolute value of companion PIDK. The blue solid line represents the sum of the fit components. The dashed red line represents the signal, while the other shaded areas represent different background components. 


\section{Inputs to the time-dependent fit}

The $B_{s}^{0} \rightarrow D_{s}^{\mp} K^{ \pm}$decay rates depend on the initially produced flavour eigenstates $\left|B_{s}^{0}(t=0)\right\rangle$ and $\left|\bar{B}_{s}^{0}(t=0)\right\rangle$. The identification of the initial flavour is performed using two flavour-tagging algorithms, referred to as the opposite-side (OS) and same-side (SS) taggers [6, 7]. The tagging algorithms use a neural network trained on simulated events (SS tagger) or data (OS tagger) to compute the probability $\eta$ to assign an incorrect flavour ("mistag") to the $B_{s}^{0}$ candidate. The estimated $\eta$ is treated as a per-candidate variable in the fit. Due to variations in the properties of tagging tracks for different channels, the predicted mistag probability $\eta$ has to be calibrated using flavour specific, self-tagging, decays to represent the true mistag rate $\omega$. Both the OS and SS taggers are calibrated using a time-dependent fit to the $B_{s}^{0} \rightarrow D_{s}^{-} \pi^{+}$control channel in which $\Delta m_{s}$ is fixed to its world-average value [9]. The statistical uncertainty on $C_{f}, S_{f}$, and $S_{\bar{f}}$ scales with $1 / \sqrt{\varepsilon_{e f f}}$, defined as $\varepsilon_{e f f}=\varepsilon_{t a g}(1-2 \omega)^{2}$ where $\varepsilon_{t a g}$ is the efficiency to tag an event. Therefore, the tagging algorithms are tuned for maximum effective tagging power. The obtained tagging power is $4.98 \pm$ $0.26 \%$ with a tagging efficiency at the level of $65.69 \pm 0.23 \%$.

The fast $B_{s}^{0}-\bar{B}_{s}^{0}$ oscillations require the finite decay-time resolution of the detector to be taken into account. The per-candidate decay-time uncertainty $\sigma_{t}$ is calibrated using prompt $D_{s}^{-}$mesons

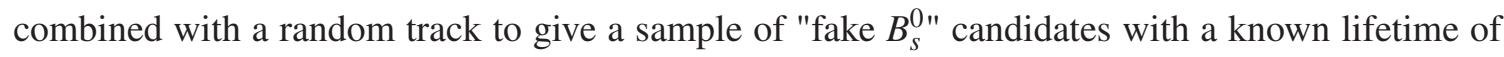
zero. The effective decay-time resolution depends on the per-candidate decay-time uncertainty as $\sigma\left(\sigma_{t}\right)=10.3 \mathrm{fs}+1.28 \sigma_{t}$ resulting in an average resolution at the level of $56 \mathrm{fs}$.

The decay-time of the $B_{s}^{0}$ mesons is distorted by several requirements which are applied in the signal selection. Non-negligible correlations between the $C P$ observables and the acceptance of the selection do not allow the latter to float in the nominal fit. The acceptance is fixed based on information from the $B_{s}^{0} \rightarrow D_{s}^{-} \pi^{+}$fit and corrected by the acceptance ratio of $B_{s}^{0} \rightarrow D_{s}^{\mp} K^{ \pm}$ and $B_{s}^{0} \rightarrow D_{s}^{-} \pi^{+}$found in simulation, where the simulation samples are weighted to match the kinematic distributions observed in data.

\section{Decay-time fit}

The determination of the $C P$ parameters is performed using an unbinned maximum likelihood fit with statistically subtracted background using the sPlot technique [8]. Therefore, only the signal decay-time distribution is described. The following parameters are fixed in the decay-time fit: $\begin{array}{lll}\Gamma_{s}=(0.6643 \pm 0.0020) \mathrm{ps}^{-1}, & \Delta \Gamma_{s}=(0.083 \pm 0.006) \mathrm{ps}^{-1}, & A_{\operatorname{det}}(K \pi)=(1 \pm 1) \%, \\ \Delta m_{s}=(17.757 \pm 0.021) \mathrm{ps}^{-1}, & \rho\left(\Gamma_{s}, \Delta \Gamma_{s}\right)=-0.239, & A_{\text {prod }}\left(B_{s}^{0}\right)=(1.1 \pm 2.7) \%,\end{array}$ based on HFAG [9] world average of $B_{s}^{0}$ oscillation frequency and decay widths, and LHCb measurements of the $B_{s}^{0}$ production asymmetry [10] and the $K^{+} \pi^{-}$detection asymmetry [11]. The quoted uncertainties of fixed parameters are used later in the systematic studies. The tagging calibration parameters are constrained to the values obtained from the control channel. The decay-time $\mathrm{PDF}$ is convolved with a single Gaussian representing the per-candidate decay-time resolution, and multiplied by the decay-time acceptance. The $C P$ violating observables are given in Table 1 , and the decay-time fit to the $B_{s}^{0} \rightarrow D_{s}^{\mp} K^{ \pm}$candidates is shown in Fig. 2.

In the measurement the following sources of systematic uncertainties are found: uncertainties from the fixed parameters $\Delta m_{s}, \Gamma_{s}$, and $\Delta \Gamma_{s}$, uncertainties from the limited knowledge of the 


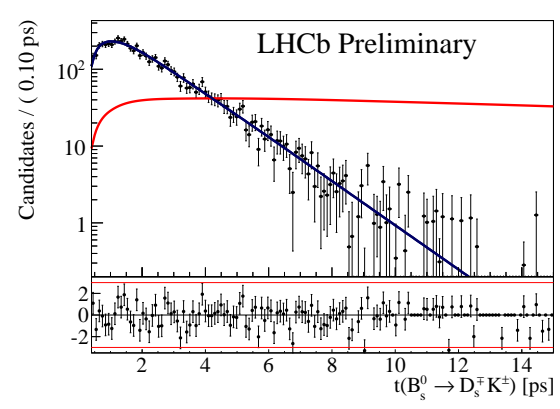

Figure 2: Result of the decay-time fit to the $B_{s}^{0} \rightarrow D_{s}^{\mp} K^{ \pm}$candidates.

Table 1: Fitted values of the $C P$ observables to the $B_{s}^{0} \rightarrow D_{s}^{\mp} K^{ \pm}$decay-time distribution where the first uncertainty is statistical, the second is systematic.

\begin{tabular}{cr}
\hline Parameter & \multicolumn{1}{c}{ Value } \\
\hline$C_{f}$ & $0.735 \pm 0.142 \pm 0.048$ \\
$A_{f}^{\Delta \Gamma}$ & $0.395 \pm 0.277 \pm 0.122$ \\
$A_{\bar{f}}^{\Delta \Gamma}$ & $0.314 \pm 0.274 \pm 0.107$ \\
$S_{f}$ & $-0.518 \pm 0.202 \pm 0.073$ \\
$S_{\bar{f}}$ & $-0.496 \pm 0.197 \pm 0.071$
\end{tabular}

detection asymmetry, the decay time resolution and acceptances and due to ignoring the correlations among observables. In addition, the sensitivity of the closure test performed to the fully simulated signal events is assigned as a systematic uncertainty. Since the acceptance parameters are determined from the fit to $B_{s}^{0} \rightarrow D_{s}^{-} \pi^{+}$candidates, where $\Gamma_{s}$ and $\Delta \Gamma_{s}$ are fixed, the systematic uncertainty on the acceptance is strongly anti-correlated with $\Gamma_{s}$ and $\Delta \Gamma_{s}$. The correlations between them are taken into account and quoted together. The summary of systematic uncertainties described as a fraction of statistical uncertainties are shown in Table 2.

Table 2: Total systematic uncertainties, relative to the statistical uncertainty.

\begin{tabular}{lccccc}
\hline Parameter & $C_{f}$ & $A_{f}^{\Delta \Gamma}$ & $A_{\bar{f}}^{\Delta \Gamma}$ & $S_{f}$ & $S_{\bar{f}}$ \\
\hline Detection asymmetry & 0.01 & 0.23 & 0.26 & 0.02 & 0.03 \\
$\Delta m_{s}$ & 0.06 & 0.01 & 0.01 & 0.17 & 0.18 \\
Tagging and resolution & 0.15 & 0.06 & 0.06 & 0.22 & 0.16 \\
Correlation among observables & 0.27 & 0.25 & 0.18 & 0.20 & 0.23 \\
Closure test & 0.12 & 0.19 & 0.19 & 0.12 & 0.12 \\
Acceptance, $\Gamma_{s}, \Delta \Gamma_{s}$ & 0.07 & 0.19 & 0.06 & 0.01 & 0.02 \\
\hline Total & 0.34 & 0.44 & 0.39 & 0.36 & 0.36
\end{tabular}

\section{Interpretation}

The measurement of the $C P$-sensitive parameters is interpreted in terms of $\gamma-2 \beta_{s}$ and subsequently $\gamma$. The value of $\beta_{s}$ is constrained to the LHCb measurement, $\phi_{s}=(-0.01 \pm 0.039) \mathrm{rad}$ 
[12]. This assumes that penguin pollution and BSM contributions are negligible, which is a good approximation at the present statistical sensitivity. The resulting confidence intervals are:

$$
\begin{array}{llll}
\gamma=\left(127_{-22}^{+17}\right)^{\circ}, & \delta=\left(358_{-16}^{+15}\right)^{\circ}, & r_{D s K}=0.37_{-0.09}^{+0.10}, & (68.3 \% \mathrm{CL}) \\
\gamma=\left(127_{-50}^{+33}\right)^{\circ}, & \delta=\left(358_{-33}^{+31}\right)^{\circ}, & r_{D s K}=0.37_{-0.19}^{+0.19}, & (95.4 \% \mathrm{CL})
\end{array}
$$

where the intervals for the angles are expressed modulo $180^{\circ}$. The quoted uncertainties are the sum in quadrature of the statistical and systematic components. The results are shown in Fig. 3.
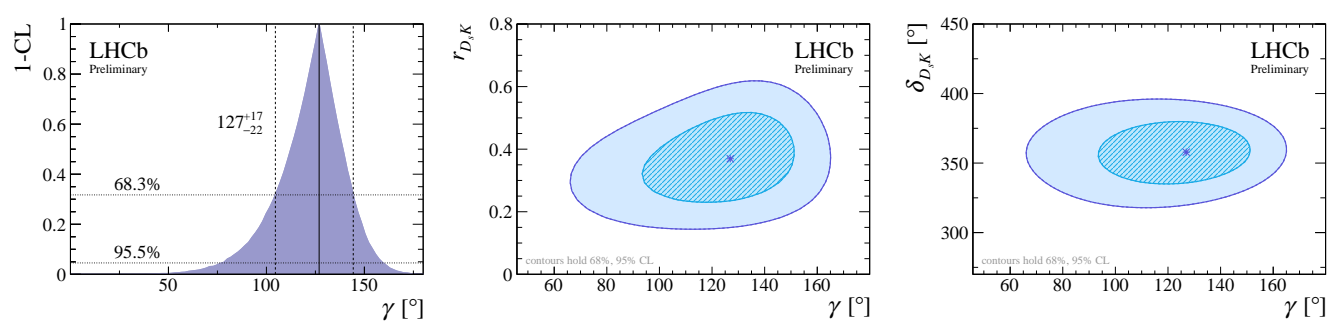

Figure 3: Graph showing 1-CL for $\gamma$, together with the central value and the $68.3 \%$ CL interval as obtained from the frequentist method [13]. Profile likelihood contours of $r_{D s K}$ vs. $\gamma$ (middle), and $\delta$ vs. $\gamma$ (right). The contours are the $1 \sigma(2 \sigma)$ profile likelihood contours, where $\Delta \chi^{2}=1\left(\Delta \chi^{2}=4\right)$, corresponding to $39 \% \mathrm{CL}$ $(86 \% \mathrm{CL})$ in Gaussian approximation. The markers denote the best-fit values.

\section{References}

[1] N. Cabibbo, Phys. Rev. Lett. 10 (1963) 531.

[2] M. Kobayashi and T. Maskawa, Prog. Theor. Phys. 49 (1973) 652.

[3] R. Fleischer, Nucl. Phys. B671 (2003) 459.

[4] J. Brod, J. Zupan, JHEP 1401 (2014) 051.

[5] LHCb collaboration, A. A. Alves Jr. et al., JINST 3 (2008) S08005.

[6] LHCb collaboration, R. Aaij et al., Eur. Phys. J. C72 (2012) 2022, arXiv:1202.4979.

[7] LHCb collaboration, R. Aaij et al., JINST 11 (2016) P05010, arXiv:1602.07252

[8] M. Pivk and F. R. Le Diberder, Nucl. Instrum. Meth. A555 (2005) 356.

[9] Heavy Flavor Averaging Group, http://www.slac.stanford.edu/xorg/hfag/.

[10] LHCb collaboration, R. Aaij et al., Phys. Lett. B739 (2014) 218, arXiv:1408.0275.

[11] LHCb collaboration, R. Aaij et al., JHEP 07 (2014) 041, arXiv:1405.2797.

[12] LHCb collaboration, R. Aaij et al., Phys. Rev. Lett. 114 (2015) 041801, arXiv:1411.3104.

[13] LHCb collaboration, R. Aaij et al., JHEP 12 (2016) 087, arXiv:1611.03076. 